\title{
COOLING OF THE CRUST IN THE NEUTRON STAR LOW-MASS X-RAY BINARY MXB 1659-29
}

\author{
Edward M. Cackett, ${ }^{1,2}$ Rudy Wijnands, ${ }^{3}$ Jon M. Miller, ${ }^{1}$ Edward F. Brown, ${ }^{4}$ And Nathalie Degenaar ${ }^{3}$ \\ Received 2008 June 6; accepted 2008 September 24; published 2008 October 14
}

\begin{abstract}
In quasi-persistent neutron star transients, long outbursts cause the neutron star crust to be heated out of thermal equilibrium with the rest of the star. During quiescence, the crust then cools back down. Such crustal cooling has been observed in two quasi-persistent sources: KS 1731-260 and MXB 1659-29. Here we present an additional Chandra observation of MXB 1659-29 in quiescence, which extends the baseline of monitoring to $6.6 \mathrm{yr}$ after the end of the outburst. This new observation strongly suggests that the crust has thermally relaxed, with the temperature remaining consistent over 1000 days. Fitting the temperature cooling curve with an exponential plus constant model we determine an $e$-folding timescale of $465 \pm 25$ days, with the crust cooling to a constant surface temperature of $k T_{\text {eff }}^{\infty}=54 \pm 2 \mathrm{eV}$ (assuming $D=10 \mathrm{kpc}$ ). From this, we infer a core temperature in the range $(3.5-8.3) \times 10^{7} \mathrm{~K}$ (assuming $D=10 \mathrm{kpc}$ ), with the uncertainty due to the surface composition. Importantly, we tested two neutron star atmosphere models as well as a blackbody model, and found that the thermal relaxation time of the crust is independent of the chosen model and the assumed distance.
\end{abstract}

Subject headings: stars: neutron - X-rays: binaries — X-rays: individual (MXB 1659-29)

\section{INTRODUCTION}

Many low-mass X-ray binaries (LMXBs) are transient, spending the majority of their time in a quiescent state with very low levels of accretion and a small fraction of their time in outburst where the mass accretion rate (and hence X-ray luminosity) increases significantly. For neutron stars, these repeated outbursts affect the star-the compression of the crust due to accretion of matter induces electron captures, neutron emissions and pycnonuclear reactions (Haensel \& Zdunik 1990) in the crust, which in turn heat the core. Over approximately $10^{4}-10^{5} \mathrm{yr}$ a steady state is reached, in which this deep crustal heating during outburst is balanced by cooling during quiescence (Brown et al. 1998).

Typically outbursts in these neutron star X-ray transients only last weeks to months; however, in the so-called quasipersistent transients, outbursts last several years. In these objects enough heat is imparted to the neutron star crust that it gets heated significantly out of thermal equilibrium with the rest of the star, which is not the case for the majority of normal X-ray transients. Therefore, in these quasi-persistent sources, once the source returns to quiescence the crust cools down into thermal equilibrium with the core over an appreciable timescale (e.g., Rutledge et al. 2002b). So far, crustal cooling curves have been observed in two such sources: MXB 1659-29 and KS 1731-260 (Wijnands et al. 2001, 2003, 2004; Cackett et al. 2006). For both objects we obtained several Chandra and XMM-Newton observations over a period of approximately 4 years after the end of long outbursts. Cackett et al. (2006) found that both sources cooled down rapidly, with the cooling well fit by an exponential decay to a constant level. This was interpreted as the neutron star crust cooling down into thermal equilibrium with the rest of the star. By comparison to crustal cooling models of Rutledge et al. (2002b) the cooling curves suggest that the crusts both have a high thermal conductivity,

\footnotetext{
${ }^{1}$ Department of Astronomy, University of Michigan, 500 Church Street, Ann Arbor, MI 48109-1042; ecackett@umich.edu.

${ }^{2}$ Dean McLaughlin Postdoctoral Fellow.

3 Astronomical Institute "Anton Pannekoek," University of Amsterdam, Kruislaan 403, 1098 SJ, Amsterdam, Netherlands.

${ }^{4}$ Department of Physics and Astronomy and the Joint Institute for Nuclear Physics, Michigan State University, East Lansing, MI 48824.
}

and the cores may require enhanced levels of neutrino emission. These observations motivated further theoretical study of crustal cooling, and models calculated by Shternin et al. (2007) for KS 1731-260 also have a best fit with a high thermal conductivity crust, but may not require enhanced neutrino emission in the core. In this Letter, we present an additional Chandra observation of MXB 1659-29, extending the quiescent monitoring by $2.8 \mathrm{yr}$ to now cover $6.6 \mathrm{yr}$ after the end of the outburst. In $\S 2$ we detail the data analysis and present the results, while in $\S 3$ we discuss the cooling curve and implications for the neutron star structure.

\section{ANALYSIS AND RESULTS}

The neutron star low-mass X-ray binary MXB 1659-29 was in outburst for about $2.5 \mathrm{yr}$, with the source going into quiescence in 2001 September. Since then, the source had been observed 6 times (5 with Chandra, 1 with XMM-Newton; see Wijnands et al. 2003, 2004; Cackett et al. 2006). On 2008 April 27 (MJD 54583.8) a 7th quiescent observation was performed-we observed MXB 1659-29 for approximately 28 ks with the Chandra ACIS-S (ObsID 8984). The source was at the default aim point on the S3 chip and was observed using the standard FAINT data mode. These data were reduced using the latest Chandra software (CIAO ver. 4.0) and calibration databases (CALDB ver. 3.4.3). No background flares were observed, so all data is used. A circular region centered on MXB 1659-29 with a radius of $3^{\prime \prime}$ was used to extract the source spectrum. For the background spectrum we used an annulus centered on the source with an inner radius of 7 " and an outer radius of 22". The source and background spectra were extracted using the CIAO tool psextract, with the correct $r m f$ and arf created using the mkacis rmf and mkarf tools.

MXB 1659-29 is seen to exhibit X-ray eclipses and dips, with an orbital period of $\sim 7.1 \mathrm{hr}$ and an eclipse length of $900 \mathrm{~s}$ (Cominsky \& Wood 1984, 1989; Oosterbroek et al. 2001). During the eclipses we do not expect to detect the source and in the very first Chandra observation of MXB 1659-29 in quiescence, the eclipse was clearly seen in the light curve (Wijnands et al. 2003). Using the ephemeris of Oosterbroek et al. (2001) we determined that only one eclipse occurred during our observation and we therefore reduced the exposure time in the spectrum by $900 \mathrm{~s}$ to account 
TABLE 1

Seectral Fitting Parameters

\begin{tabular}{|c|c|c|c|c|c|c|c|}
\hline Parameter & $\begin{array}{c}2688 \\
(\mathrm{CXO})\end{array}$ & $\begin{array}{c}3794 \\
(\mathrm{CXO})\end{array}$ & $\begin{array}{c}0153190101 \\
(\mathrm{XMM})\end{array}$ & $\begin{array}{c}3795 \\
(\mathrm{CXO})\end{array}$ & $\begin{array}{c}5469 \\
(\mathrm{CXO})\end{array}$ & $\begin{array}{c}6337 \\
(\mathrm{CXO})\end{array}$ & $\begin{array}{c}8984 \\
(\mathrm{CXO})\end{array}$ \\
\hline MJD & 52197.8 & 52563.2 & 52712.2 & 52768.9 & 53560.0 & 53576.7 & 54583.8 \\
\hline \multicolumn{8}{|c|}{$\mathrm{nsa}, D=10 \mathrm{kpc}$} \\
\hline $\begin{array}{l}N_{\mathrm{H}}\left(10^{21} \mathrm{~cm}^{-2}\right) \\
k T_{\text {eff }}^{\infty}(\mathrm{eV}) \\
F_{\text {bol }}\left(10^{-14} \mathrm{erg} \mathrm{cm}^{-2} \mathrm{~s}^{-1}\right) \\
\end{array}$ & $\begin{array}{c}121 \pm 1 \\
41 \pm 2 \\
\end{array}$ & $\begin{array}{l}85 \pm 1 \\
10 \pm 1 \\
\end{array}$ & $\begin{array}{c}77 \pm 1 \\
6.6 \pm 0.3 \\
\end{array}$ & $\begin{array}{c}2.0 \pm 0.2 \\
73 \pm 1 \\
5.1 \pm 0.3 \\
\end{array}$ & $\begin{array}{c}58 \pm 2 \\
2.0 \pm 0.3\end{array}$ & $\begin{array}{c}54 \pm 3 \\
1.5 \pm 0.3 \\
\end{array}$ & $\begin{array}{c}56 \pm 2 \\
1.8 \pm 0.3 \\
\end{array}$ \\
\hline \multicolumn{8}{|c|}{$\mathrm{nsa}, D=5 \mathrm{kpc}$} \\
\hline $\begin{array}{l}N_{\mathrm{H}}\left(10^{21} \mathrm{~cm}^{-2}\right) \\
k T_{\text {eff }}^{\infty}(\mathrm{eV}) \\
F_{\text {bol }}\left(10^{-14} \mathrm{erg} \mathrm{cm}^{-2} \mathrm{~s}^{-1}\right)\end{array}$ & $\begin{array}{l}95 \pm 1 \\
59 \pm 2 \\
\end{array}$ & $\begin{array}{l}69 \pm 1 \\
16 \pm 1 \\
\end{array}$ & $\begin{array}{l}63 \pm 1 \\
11 \pm 1 \\
\end{array}$ & $\begin{array}{c}2.9 \pm 0.1 \\
59 \pm 1 \\
9.0 \pm 0.6 \\
\end{array}$ & $\begin{array}{c}48 \pm 1 \\
3.7 \pm 0.4\end{array}$ & $\begin{array}{c}45 \pm 2 \\
2.9 \pm 0.5\end{array}$ & $\begin{array}{c}47 \pm 2 \\
3.5 \pm 0.5\end{array}$ \\
\hline \multicolumn{8}{|c|}{$\mathrm{nsa}, D=13 \mathrm{kpc}$} \\
\hline $\begin{array}{l}N_{\mathrm{H}}\left(10^{21} \mathrm{~cm}^{-2}\right) \\
k T_{\text {eff }}^{\infty}(\mathrm{eV}) \\
F_{\text {bol }}\left(10^{-14} \mathrm{erg} \mathrm{cm}^{-2} \mathrm{~s}^{-1}\right)\end{array}$ & $\begin{array}{c}133 \pm 1 \\
34 \pm 1\end{array}$ & $\begin{array}{c}93 \pm 1 \\
8.1 \pm 0.5\end{array}$ & $\begin{array}{c}84 \pm 1 \\
5.4 \pm 0.2\end{array}$ & $\begin{array}{c}1.6 \pm 0.1 \\
79 \pm 2 \\
4.1 \pm 0.3\end{array}$ & $\begin{array}{c}62 \pm 2 \\
1.6 \pm 0.2\end{array}$ & $\begin{array}{c}57 \pm 3 \\
1.2 \pm 0.2\end{array}$ & $\begin{array}{c}61 \pm 2 \\
1.5 \pm 0.2\end{array}$ \\
\hline \multicolumn{8}{|c|}{ nsatmos, $D=10 \mathrm{kpc}$} \\
\hline $\begin{array}{l}N_{\mathrm{H}}\left(10^{21} \mathrm{~cm}^{-2}\right) \\
k T_{\text {eff }}^{\infty}(\mathrm{eV}) \\
F_{\text {bol }}\left(10^{-14} \mathrm{erg} \mathrm{cm}^{-2} \mathrm{~s}^{-1}\right) \\
\end{array}$ & $\begin{array}{c}121 \pm 1 \\
39 \pm 1 \\
\end{array}$ & $\begin{array}{c}86 \pm 1 \\
9.8 \pm 0.5 \\
\end{array}$ & $\begin{array}{c}78 \pm 1 \\
6.6 \pm 0.2\end{array}$ & $\begin{array}{c}2.0 \pm 0.1 \\
73 \pm 1 \\
5.1 \pm 0.4 \\
\end{array}$ & $\begin{array}{c}57 \pm 2 \\
2.0 \pm 0.3\end{array}$ & $\begin{array}{c}54 \pm 3 \\
1.5 \pm 0.3 \\
\end{array}$ & $\begin{array}{c}57 \pm 2 \\
1.9 \pm 0.3 \\
\end{array}$ \\
\hline \multicolumn{8}{|c|}{ bbodyrad } \\
\hline $\begin{array}{l}N_{\mathrm{H}}\left(10^{21} \mathrm{~cm}^{-2}\right) \\
\text { Normalization } \\
k T_{\text {eff }}^{\infty}(\mathrm{eV}) \\
F_{\text {bol }}\left(10^{-14} \mathrm{erg} \mathrm{cm}^{-2} \mathrm{~s}^{-1}\right)\end{array}$ & $\begin{aligned} 300 & \pm 11 \\
29 & \pm 4\end{aligned}$ & $\begin{array}{c}213 \pm 6 \\
7.2 \pm 0.9\end{array}$ & $\begin{array}{c}193 \pm 5 \\
4.9 \pm 0.5\end{array}$ & $\begin{array}{l}1.6 \pm 0.2 \\
3.3_{-0.5}^{+0.9} \\
184 \pm 5 \\
4.0 \pm 0.5\end{array}$ & $\begin{array}{c}148 \pm 5 \\
1.7 \pm 0.2\end{array}$ & $\begin{array}{c}140 \pm 7 \\
1.3 \pm 0.3\end{array}$ & $\begin{array}{c}147 \pm 5 \\
1.6 \pm 0.2\end{array}$ \\
\hline
\end{tabular}

Notes.-All uncertainties are $1 \sigma$. The mass and radius are fixed in all fits to $1.4 M_{\odot}$ and $10 \mathrm{~km}$, respectively. In the nsatmos fits the normalization is fixed at 1.0. ObsIDs of each observation are indicated at the top, with CXO (Chandra) and XMM (XMM-Newton) denoting the observatory.

${ }^{a}$ Bolometric flux calculated from the model over the $0.01-100 \mathrm{keV}$ range.

${ }^{\mathrm{b}}$ Normalization for the blackbody model is $(R / \mathrm{D} 10)^{2} . R$ is the emitting radius $(\mathrm{km})$ and $\mathrm{D} 10$ is $(D / 10 \mathrm{kpc})$.

for the fact that we would not receive any counts during the eclipse. After this correction, the background-subtracted net count rate in the $0.5-10 \mathrm{keV}$ band is $(1.0 \pm 0.2) \times 10^{-3}$ counts $\mathrm{s}^{-1}$, with the corrected exposure time $26.7 \mathrm{ks}$.

\subsection{Spectral Fitting}

In this analysis we fit this latest Chandra spectrum simultaneously with the previous 5 Chandra and 1 XMM-Newton observations of MXB 1659-29 in quiescence (we do not create new spectra of these previous observations here; see Cackett et al. 2006). For spectral fitting we used XSPEC (ver. 11; Arnaud 1996). The spectra were left unbinned due to the low number of counts and the W-statistic (Wachter et al. 1979) was used in all fits.

Neutron star atmosphere spectra deviate from that of a simple blackbody as they have a slightly harder tail due to the strong frequency dependence of opacity for free-free absorption (Zavlin et al. 1996). This difference means that when a blackbody model is fitted to the X-ray spectra of quiescent neutron stars a significantly higher surface temperature and an unrealistically small emitting radius are determined (e.g., Rutledge et al. 1999). The spectra were therefore fitted with an absorbed neutron star atmosphere model. There are currently a variety of neutron star atmosphere models available for the pure hydrogen, low-magnetic field case that is relevant here. In Cackett et al. (2006) we used the nsa model (Pavlov et al. 1991; Zavlin et al. 1996), which we also use here. In order to test the model dependence of the cooling curves we chose to also fit the nsatmos model (Heinke et al. 2006). Finally, for completeness, we also fit a simple blackbody model (bbodyrad).
In the neutron star atmosphere fits, we fixed the mass and radius at the canonical values of $1.4 M_{\odot}$ and $10 \mathrm{~km}$ as the data is not of high enough quality to independently determine these parameters. The normalization in the nsa model is defined as $1 / D^{2}$, where $D$ is the distance to the object in pc. In the nsatmos model there is also a distance parameter, as well as a separate normalization, $K$, which corresponds to the fraction of the neutron star surface that is emitting. We fix $K=1$ in all our fits with nsatmos. In Cackett et al. (2006) we fixed $D=10 \mathrm{kpc}$ to reduce the number of free parameters in the fit given the low number of counts in the majority of the spectra. If the distance is left as a free parameter the uncertainty in the parameters are dominated by the uncertainty in the distance. The distance to MXB $1659-29$ is estimated to be $10-13 \mathrm{kpc}$ (Muno et al. 2001; Oosterbroek et al. 2001) from using type I X-ray bursts. Here we investigated the dependence of the cooling timescale on the assumed distance by performing spectral fits with $D=5,10$, and $13 \mathrm{kpc}$. Wijnands et al. (2004) found that when assuming 3 different distances to MXB 1659-29 there was little effect on the $e$-folding timescale.

For the interstellar photoelectric absorption we used the phabs model, and this parameter is tied between all the observations. Therefore in the spectral fits the free parameters are the absorbing column and the effective temperature. Finally, for the one XMM-Newton observation we tie the parameters between the MOS1, MOS2, and pn spectra. Results of the spectral fitting are given in Table 1 . Note that we quote $k T_{\text {eff }}^{\infty}$, the effective surface temperature for an observer at infinity. The bolometric flux was estimated by extrapolating the unabsorbed model over the $0.01-100 \mathrm{keV}$ range. All uncertainties 


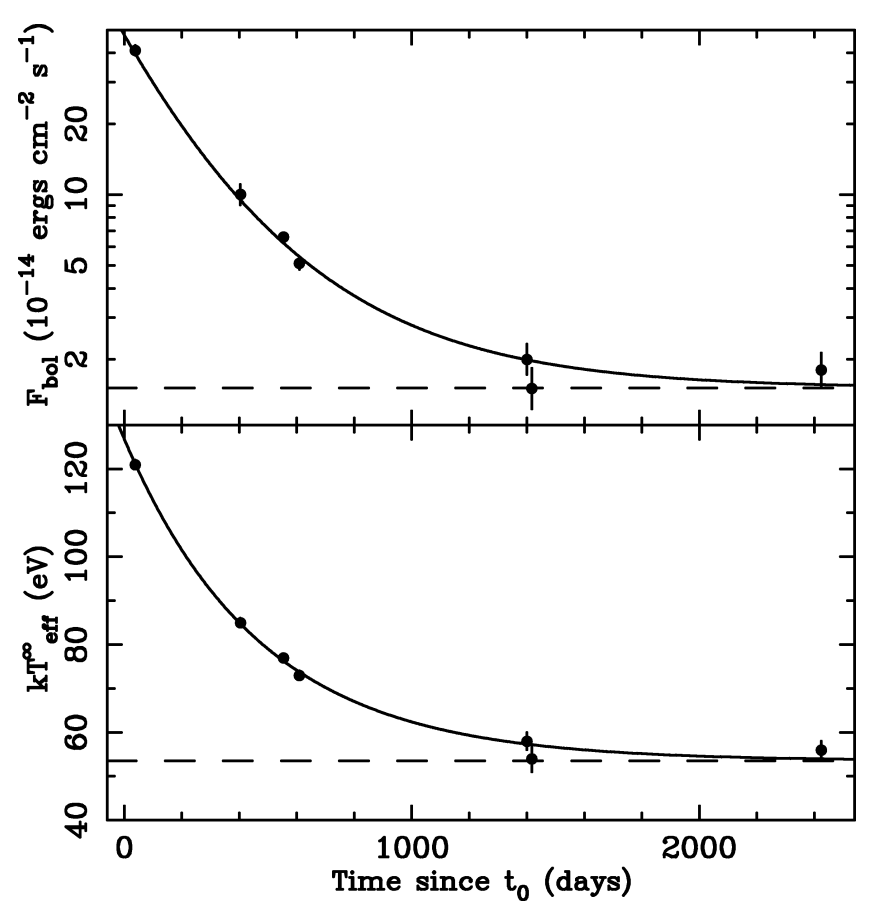

FIG. 1.-Flux (top) and temperature (bottom) cooling curves for MXB 1659-29. The best-fitting model to the temperatures comprising an exponential decay to a constant level is shown (solid line), where the constant offset is shown with a dashed line. The flux cooling curve is then calculated from the fit to the temperatures. The time $t_{0}=52159.5$ (MJD) is the last day when the source was seen to be active. Error bars are $1 \sigma$. Data points are from the nsa fits with the distance fixed at $10 \mathrm{kpc}$.

quoted and plotted in this Letter are $1 \sigma$. We note that in Cackett et al. (2006) the uncertainties quoted on the spectral fits and plotted on the figures are at the $90 \%$ level of confidence not $1 \sigma$ as is incorrectly written in the text.

All the spectral fits show that the temperature and flux of this new observation has remained consistent with the last two observations performed approximately 1000 days previously, regardless of the model used, or distance assumed, in the fitting. The temperature and flux determined from the nsa fits with $D=10 \mathrm{kpc}$ is shown in Figure 1. We found that there is very good agreement between the effective temperature determined by the nsa and nsatmos fits. In addition, we found that the temperatures and fluxes from the neutron star atmosphere fits are just shifted up or down in a systematic way depending on the assumed distance. The temperatures determined from the bbodyrad fits are significantly higher than the neutron star atmosphere temperatures. Moreover, if a distance of $10 \mathrm{kpc}$ is assumed, the normalization implies an emitting radius of $\sim 2$ $\mathrm{km}$, much smaller than is realistic if the entire surface is emitting. Both high temperatures and small emitting radii are normally found when fitting a blackbody model to quiescent neutron stars (e.g., Rutledge et al. 1999).

\subsection{Cooling Curves}

The results of the spectral fitting (Table 1) show that after the initial cooling the temperature is consistent with being constant over the last 1000 days. In Cackett et al. (2006) we found that the cooling curves for both MXB 1659-29 and KS 1731-260 could be well fit by an exponential that decays to a constant level, interpreted as the neutron star crust cooling back into thermal equilibrium with the core. With the addition of the new observation of MXB 1659-29 we fitted the cooling
TABLE 2

Cooling Curve Parameters from Fits to the Temperatures

\begin{tabular}{lcccc}
\hline \hline & \multicolumn{4}{c}{$\mathrm{nsa}$} \\
\cline { 2 - 4 } Parameter & $D=10 \mathrm{kpc}$ & $D=5 \mathrm{kpc}$ & $D=13 \mathrm{kpc}$ & bbodyrad \\
\hline$a(\mathrm{eV})$ & $73 \pm 2$ & $55 \pm 1$ & $81 \pm 2$ & $175 \pm 12$ \\
$b$ (days) & $465 \pm 25$ & $483 \pm 30$ & $473 \pm 23$ & $435 \pm 44$ \\
$c(\mathrm{eV})$ & $54 \pm 2$ & $45 \pm 1$ & $58 \pm 2$ & $142 \pm 4$ \\
$\chi_{\nu}^{2}$ & 0.8 & 1.5 & 1.4 & 0.7 \\
$P_{\chi}$ & 0.52 & 0.18 & 0.21 & 0.61 \\
\hline
\end{tabular}

Notes. - Here $a=$ normalization, $b=e$-folding time, and $c=$ constant. The model fit to the cooling curves is of the form $y(t)=a \exp [-(t-$ $\left.\left.t_{0}\right) / b\right]+c$, where $t_{0}=52159.5$. All uncertainties are $1 \sigma$.

curve again with the same model of the form $y(t)=$ $a \exp \left[-\left(t-t_{0}\right) / b\right]+c$. We set $t_{0}$ to midday on the last day that the source was observed to be active, MJD 52159.5. This model was fit to the temperatures determined by the nsa spectral fits (for all assumed distances) as well as to the results of the blackbody fits, allowing us to test the model dependence of the cooling curves. We do not fit curves to the nsatmos results as they so closely match the nsa results. We only fit the cooling curve to the temperatures, as from this fit one can directly calculate the flux via $F=\sigma T_{\text {eff }}^{\infty 4}\left(R_{a} / D\right)^{2}$, where $R_{a}$ is the apparent radius for an observer at infinity.

The results of these exponential decay plus constant fits are given in Table 2, and we show the temperature and flux cooling curves for the nsa fits (with $D=10 \mathrm{kpc}$ ) in Figure 1 . There is excellent agreement between the $e$-folding timescales, $b$, when using results from different spectral models and assuming different distances, demonstrating that the cooling timescale is robust. For comparison, the cooling curve values for $\mathrm{KS}$ $1731-260$ values are $a=40 \pm 3 \mathrm{eV}, b=305 \pm 47$ days, $c=70.2 \pm 1.2 \mathrm{eV}$ fitting the temperatures, assuming $D=$ $7 \mathrm{kpc}$. These are updated from Cackett et al. (2006) using the correct $1 \sigma$ uncertainties, and rectifying a minor error in the fitting code. Note, however, that these values remain consistent with those quoted in Cackett et al. (2006).

The exponential plus constant model fits the data well with the reduced $\chi^{2}$ values all close to 1 , and the $\chi^{2}$ probabilities, $P_{\chi}$, all close to 0.5 , as expected for a good fit. However, the mathematical solution for the flux from a cooling thin layer is a (possibly broken) power law (Eichler \& Cheng 1989; Piro et al. 2005), not an exponential. We therefore also attempt to fit a single power law to the data of the form $y(t)=a\left(t-t_{0}\right)^{b}$, and find that such a model does not fit the data well. However, inspection of the cooling curve indicates that the middle section of the curve does appear to follow a power law. Fitting a power law to just these middle data points (excluding the first and last observation) achieves a good fit. For fits to the temperatures from the nsa results with $D=10 \mathrm{kpc}$, we find the power-law index $b=-0.33 \pm 0.02, \chi_{\nu}^{2}=0.9$, and $P_{\chi}=0.46$. The effective temperature from the last observation is a $4.5 \sigma$ deviation from an extrapolation of this best-fitting power law. This again strongly indicates that the temperature is now remaining constant, and that the crust is thermally relaxed.

\section{DISCUSSION}

We presented a new observation of the quasi-persistent neutron star X-ray transient MXB 1659-29 in quiescence, extending the quiescent monitoring to $6.6 \mathrm{yr}$. Results from the first 6 observations showed that the source had cooled rapidly and indicated that the neutron star crust may have returned to thermal equilibrium with the core. This new observation shows 
that the neutron star temperature and flux remained consistent with the previous two Chandra observations performed approximately 1000 days before. The model dependence of the thermal relaxation timescale was investigated with 2 different neutron star atmosphere models as well as a simple blackbody model. Moreover, we assumed 3 different distances to MXB 1659-29. The $e$-folding timescales of the cooling curves from all the spectral fits are consistent with each other, demonstrating the robustness of the measurement. The results are consistent with fits to the first 6 observations (Cackett et al. 2006).

With the crust thermally relaxed, we can compute the core temperature (here we assume $D=10 \mathrm{kpc}$ ). We integrate the thermal structure equation in the neutron star envelope, following the calculation in Brown et al. (2002). The inferred core temperature is relatively insensitive to the mass of the neutron star (the proper $T_{\text {eff }}$ increases with redshift for a fixed $T_{\text {eff }}^{\infty}$, but the surface layer becomes thinner with increasing $g$ and reduces the rise in temperature in the envelope). There is a significant uncertainty resulting from the depth of the light element layer, however (Brown et al. 2002). We find the inferred core temperature to range from $3.5 \times 10^{7} \mathrm{~K}$, for a deep He layer (column of $10^{8} \mathrm{~g} \mathrm{~cm}^{-2}$ ) overlaying a pure Fe layer to $8.3 \times 10^{7} \mathrm{~K}$ for an shallow He layer (column of $10^{4} \mathrm{~g} \mathrm{~cm}^{-2}$ ) overlaying a layer of heavy rp-process ashes. We estimate the time-averaged mass accretion rate for the system to be $7 \times 10^{-11} M_{\odot} \mathrm{yr}^{-1}$, based on the known outburst behavior of 2 outbursts lasting approximately $2.5 \mathrm{yr}$, with a quiescent period of $21 \mathrm{yr}$. We estimated the fraction of Eddington luminosity by calculating the ratio of the average persistent outburst flux to the peak type I X-ray burst flux (taken from Wijnands et al. 2002). From this, we estimated the time-averaged crust nuclear heating, assuming the heat deposited in the crust is $1.5 \mathrm{MeV}$ per nucleon (see Brown et al. 1998; Rutledge et al. 2002a), to be $\sim 6 \times 10^{33} \mathrm{ergs} \mathrm{s}^{-1}$, for a distance of $10 \mathrm{kpc}$.

As noted previously (Cackett et al. 2006; Heinke et al. 2007), even for the highest core temperature compatible with $T_{\text {eff }}^{\infty}$, the neutrino luminosity resulting from modified Urca cooling (for a review, see Yakovlev \& Pethick 2004) would still be a factor of $\approx 30$ less than the time-averaged crust nuclear heating. As a check on whether there is a need for enhanced cooling, we also computed the neutrino cooling according to the "minimal cooling model" (Page et al. 2004), which includes the pair breaking and formation (PBF) neutrino emissivity, but with the ${ }^{1} S_{0}$ channel suppressed following Steiner \& Reddy (2008). In this case the PBF neutrino luminosity from neutrons in the
${ }^{3} P_{2}$ state is still sufficient to balance the time-averaged nuclear heating, if the core temperature is in the upper half of the range given above. Given the uncertainty in the depth of the light element envelope and the superfluid critical temperatures, we cannot exclude that the neutrino emission is solely due to standard cooling processes.

The exponential plus constant cooling curve fits provide a measure of the thermal relaxation time of the crust. This relaxation time depends on the crust composition and lattice structure (Rutledge et al. 2002b; Shternin et al. 2007), on the crust thickness and hence surface gravity of the neutron star (Lattimer et al. 1994), and on the distribution of heat sources (Shternin et al. 2007; Horowitz et al. 2008b; E. F. Brown \& A. Cumming, in preparation). Shternin et al. (2007) showed that the cooling timescale in KS $1731-260$ was best fit by having a high thermal conductivity in the crust, as if it were composed of a locally pure lattice. This matches molecular dynamics simulations (Horowitz et al. 2007, 2008a), which find that the dense crust plasma does indeed freeze into an ordered lattice with a high thermal conductivity. As in KS 1731-260, our fits to the cooling of MXB 1659-29 are again consistent with such an ordered, low-impurity crust. Shternin et al. (2007) noted that the crust may not have completely thermally relaxed; we find a single power-law decay also fits the cooling curve for KS $1731-260$ well, with a power-law index $=-0.12$ \pm 0.01 and $\chi_{v}^{2}=0.2$ when fitting to the temperatures. Further observations are required to determine whether KS 1731-260 has continued to cool following a power-law decay or if it has reached a constant $k T_{\text {eff }}^{\infty}$ indicative of a thermally relaxed crust. Shternin et al. (2007) note that the cooling of KS 1731-260 can be fit without invoking enhanced neutrino emission in the core. If MXB 1659-29 does have a higher core neutrino emission than KS 1731-260 then this may imply that the neutron star in MXB 1659-29 is somewhat more massive than the one in KS 1731-260 since most equations of state allow higher levels of neutrino emission with increasing central density. We note, however, that this conclusion assumes that the time-averaged mass accretion rates in both these objects have remained in a steady state.

J. M. M. gratefully acknowledges support from Chandra. E. F. B. is supported by Chandra Award TM7-8003X issued by the Chandra X-Ray Observatory Center, and by ATFP grant NNX08AG76G.

\section{REFERENCES}

Arnaud, K. A. 1996, in ASP Conf. Ser. 101, Astronomical Data Analysis Software and Systems V, ed. G. H. Jacoby \& J. Barnes (San Francisco: ASP), 17

Brown, E. F., Bildsten, L., \& Chang, P. 2002, ApJ, 574, 920

Brown, E. F., Bildsten, L., \& Rutledge, R. E. 1998, ApJ, 504, L95

Cackett, E. M., Wijnands, R., Linares, M., Miller, J. M., Homan, J., \& Lewin, W. H. G. 2006, MNRAS, 372, 479

Cominsky, L. R., \& Wood, K. S. 1984, ApJ, 283, 765

- 1989, ApJ, 337, 485

Eichler, D., \& Cheng, A. F. 1989, ApJ, 336, 360

Haensel, P., \& Zdunik, J. L. 1990, A\&A, 227, 431

Heinke, C. O., Jonker, P. G., Wijnands, R., \& Taam, R. E. 2007, ApJ, 660, 1424

Heinke, C. O., et al. 2006, ApJ, 644, 1090

Horowitz, C. J., Berry, D. K., \& Brown, E. F. 2007, Phys. Rev. E, 75, 066101

Horowitz, C. J., Caballero, O. L., \& Berry, D. K. 2008a, Phys. Rev. E, submitted (arXiv:0804.4409)

Horowitz, C. J., Dussan, H., \& Berry, D. K. 2008b, Phys. Rev. C, 77, 045807 Lattimer, J. M., van Riper, K. A., Prakash, M., \& Prakash, M. 1994, ApJ, 425, 802 Muno, M. P., Chakrabarty, D., Galloway, D. K., \& Savov, P. 2001, ApJ, 553, L157 Oosterbroek, T., et al. 2001, A\&A, 376, 532

Page, D., Lattimer, J. M., Prakash, M., \& Steiner, A. W. 2004, ApJS, 155, 623
Pavlov, G. G., Shibanov, I. A., \& Zavlin, V. E. 1991, MNRAS, 253, 193

Piro, A. L., Arras, P., \& Bildsten, L. 2005, ApJ, 628, 401

Rutledge, R. E., Bildsten, L., Brown, E. F., Pavlov, G. G., \& Zavlin, V. E. 1999, ApJ, 514, 945

2002a, ApJ, 577, 346

Rutledge, R. E., Bildsten, L., Brown, E. F., Pavlov, G. G., Zavlin, V. E., \& Ushomirsky, G. 2002b, ApJ, 580, 413

Shternin, P. S., Yakovlev, D. G., Haensel, P., \& Potekhin, A. Y. 2007, MNRAS, 382, L43

Steiner, A. W., \& Reddy, S. 2008, Phys. Rev. C, submitted (arXiv:0804.0593)

Wachter, K., Leach, R., \& Kellogg, E. 1979, ApJ, 230, 274

Wijnands, R., Homan, J., Miller, J. M., \& Lewin, W. H. G. 2004, ApJ, 606, L61

Wijnands, R., Miller, J. M., Markwardt, C., Lewin, W. H. G., \& van der Klis, M. 2001, ApJ, 560, L159

Wijnands, R., Muno, M. P., Miller, J. M., Franco, L. M., Strohmayer, T., Galloway, D., \& Chakrabarty, D. 2002, ApJ, 566, 1060

Wijnands, R., Nowak, M., Miller, J. M., Homan, J., Wachter, S., \& Lewin, W. H. G. 2003, ApJ, 594, 952

Yakovlev, D. G., \& Pethick, C. J. 2004, ARA\&A, 42, 169

Zavlin, V. E., Pavlov, G. G., \& Shibanov, Y. A. 1996, A\&A, 315, 141 